\title{
Efficient and Versatile Locomotion With Highly Compliant Legs
}

\author{
Marco Hutter, C. David Remy, Mark A. Hoepflinger, and Roland Siegwart, Fellow, IEEE
}

\begin{abstract}
Drawing inspiration from nature, this paper introduces and compares two compliant robotic legs that are able to perform precise joint torque and position control, enable passive adaption to the environment, and allow for the exploitation of natural dynamic motions. We report in detail on the design and control of both prototypes and elaborate specifically on the problem of precise foot placement during flight without the sacrifice of efficient energy storage during stance. This is achieved through an integrated design and control approach that incorporates series elastic actuation, series damping actuation, and active damping through torque control. The two legs are employed in efficient hopping/running motions for which they achieve performance similar to humans or animals. This paper is concluded by a comparison of the various design choices with respect to performance and applicability, as well as an outlook on the usage of these legs in a fully actuated quadruped.
\end{abstract}

Index Terms-Legged robot, scarlETH, series elastic actuation (SEA), torque control.

\section{INTRODUCTION}

A NIMALS and humans can exhibit astonishing levels of versatility when moving in rough and highly unstructured terrain, while at the same time, they are able to locomote with high speed and great efficiency on less demanding surfaces. We can easily climb over obstacles the size of our leg length, or move along steep slopes by carefully selecting footholds and precisely moving our limbs to keep balance at all times. On flat terrain, however, we switch to periodic gaits (such as walking or running) which are largely driven by the natural dynamics of our mechanical structure [1], [2] and are hence energetically efficient.

Over the past decades, the robotics community was able to successfully reduce the performance gap between robots and nature in terms of versatility by building on their strong background in industrial robotics. The resulting systems (often highly sophisticated humanoid robots with a large amount of degrees of freedom (DoFs) [3], [4]) are built mechanically rigid and are driven by stiff actuators with large gearbox transmissions

Manuscript received October 31, 2011; revised April 11, 2012; accepted September 16, 2012. Date of publication October 22, 2012; date of current version January 10, 2013. Recommended by Guest Editor M. Sitti. This work was supported in part by the Swiss National Science Foundation through Project 200021_119965/1, in part by the Swiss National Centre of Competence in Research, and in part by the Hans-Eggenberger-Foundation.

M. Hutter, M. A. Hoepflinger, and R. Siegwart are with the Autonomous Systems Laboratory, Swiss Federal Institute of Technology, 8006 Zurich, Switzerland (e-mail: mahutter@ethz.ch; markho@ethz.ch; rsiegwart@ethz.ch).

C. D. Remy is with the Department of Mechanical Engineering, University of Michigan, Ann Arbor, MI 48109 USA (e-mail: cdremy@umich.edu).

Color versions of one or more of the figures in this paper are available online at http://ieeexplore.ieee.org.

Digital Object Identifier 10.1109/TMECH.2012.2222430 and high-gain controllers. This design allows them to precisely follow kinematic joint trajectories, thus enabling a large variety of motion tasks. Similar systems with more than two legs were developed for static locomotion in rough terrain [5] and are able to transverse all kinds of obstacles. A recent example of research in this direction is the DARPA Learning Locomotion Challenge (see, e.g., [6] and [7]), in which several groups pushed the state of the art with respect to motion planning and execution in unstructured, yet fully known terrain. Toward the same direction, our group developed ALoF; a highly mobile quadruped [8] which is employed to study the usage of haptic feedback to estimate surface properties [9].

In stark contrast to this kinematic approach are robots that exploit their natural dynamics to improve locomotion speed and efficiency. Initiated by Raibert's seminal work on hopping robots with pneumatic pistons [10], researchers started using elastic elements to temporarily store kinetic energy and thus preserve it throughout the gait cycle. This makes locomotion efficient and enables running at high speeds. Conceptually, such robots reflect the abstract model of the spring loaded inverted pendulum (SLIP [11], [12]) that is often used to explain the energetics of running motions found in nature. Depending on the particular design, springs are integrated directly in prismatic legs [13]-[15] or into the knee and ankle joints of articulated designs [16]-[20]. In all these robots, motion emerges to a large extent passively from the mechanical dynamics and actuators are merely used to compensate for friction and impact losses.

So far, all presented systems share one substantial drawback: They either solve the versatility or the efficiency problem, but never both. Kinematically controlled robots must create the entire motion actively, which tends to make them quite inefficient. Additionally, they cannot tolerate high impact forces and are thus unsuited for highly dynamic maneuvers. And since they are unable to adapt passively to uncertainties in the environment, they require extensive (and expensive) sensor equipment to cope with unforeseen situations. Robots that exploit natural dynamics are, on the other hand, inherently hard to control and are thus gravely limited with respect to their application. Many of the existing solutions are unable to turn or stop [21], and to the best of our knowledge, solutions for robotic devices that demonstrate both, precise kinematic control as well as the exploitation of natural dynamics, are still not available.

As a step toward closing this gap, our group developed a series of compliant, highly integrated legs (see Fig. 1) that allow for precise joint torque and position control, enable passive adaption, and allow for natural dynamic motions. In this paper, we introduce, discuss, and compare two of these legs, FerrETH (first elastic running robot) and ScarlETH (series compliant articulated robotic leg). We largely extend findings of previous 


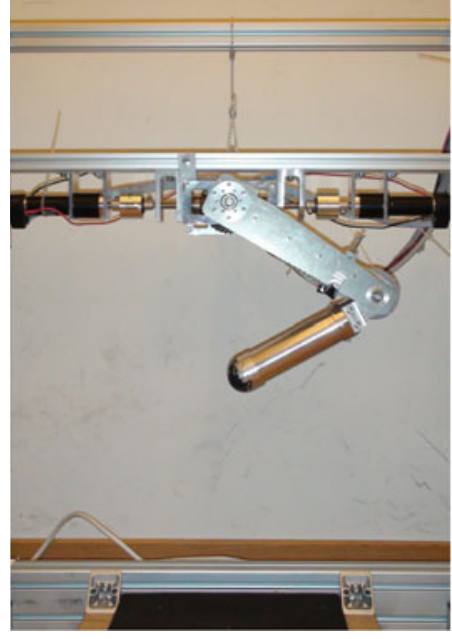

(a)

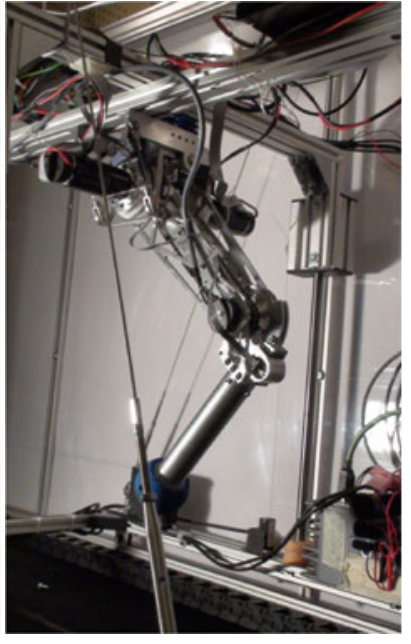

(b)
Fig. 1. Two compliant legged prototypes developed to investigate versatile and efficient locomotion. (a) FerrETH (first elastic running robot). (b) ScarlETH (series compliant articulated robotic leg).

studies with FerrETH [22], [23] and ScarlETH [24], [25] and compare them in terms of performance and applicability. We further report in detail on their design and control and elaborate specifically on how to create the necessary damping without sacrificing efficiency.

\section{SYSTEM DESIGN}

Design guidelines for legged systems that are specifically built for dynamic maneuvers can be directly adopted from their counterparts in nature. For example, to reduce the leg inertia (to allow faster joint motion and decrease losses at touchdown), segments should be built as lightweight as possible, and heavy components, such as actuators, should be placed close to the main body. Elastic elements should decouple actuators and joints in order to make the system robust against impact collisions, to allow passive adaptation, and to enable the temporary storage of energy [11]. To maximize mobility, one should aim for a large range of motion in all joints and thus tightly integrate all mechanical and electrical components. In this section, we will show how these design guidelines have been implemented in two different leg prototypes while focusing specifically on different actuation solutions based on series elastic actuation (SEA) and/or series damping actuation (SDA). A comparison of the mechanical properties and components of both legs can be found in Table I.

\section{A. FerrETH}

To compactly integrate the hip (Maxon RE-25 20-W DC motors with Maxon GP-32 1:51 planetary gearbox) and knee actuators (Maxon RE-35 90-W DC motors with Maxon GP-42 1:26 planetary gearbox) within the main body of FerrETH (see Fig. 2), a differential drive (VII) couples the DoFs for hip abduction and adduction (cf., [8]). Additionally, the knee motor (IV) is integrated in a hollow shaft going through the bevel gear for hip flexion/extension and from there connected through a miniature
TABLE I

KEy DATA OF FERRETH AND SCARLETH

\begin{tabular}{|c|c|c|}
\hline parameter & FerrETH & ScarlETH \\
\hline \multicolumn{3}{|c|}{ mechanics } \\
\hline$m_{S}$ (Shank) & 0.43 & 0.42 \\
\hline$m_{T}$ (Thigh) & 1.7 & $1.56 \mathrm{~kg}$ \\
\hline$m_{H}$ (Hip) & 1.9 & 0.56 \\
\hline$m_{\text {Payload }}$ & $\mathrm{kg}$ & 3.0 \\
\hline$\theta_{S}$ & $1.2 \mathrm{E}-3$ & $3.5 \mathrm{E}-3$ \\
\hline$\theta_{T}$ & $7.8 \mathrm{E}-3$ & $8.5 \mathrm{E}-3$ \\
\hline$l_{S}$ & 0.18 & 0.2 \\
\hline$l_{T}$ & 0.2 & 0.2 \\
\hline$s_{S}{ }^{\mathrm{a}}$ & 0.074 & 0.08 \\
\hline$s_{T}{ }^{\mathrm{a}}$ & 0.005 & 0.016 \\
\hline \multicolumn{3}{|c|}{ actuation } \\
\hline hip motors & Maxon DC RE25 (20W) & Maxon DC RE35, 90W \\
\hline knee motor & Maxon DC RE35 (90W) & Maxon DC RE35, 90W \\
\hline hip gearbox & Maxon GP32 ${ }^{b}(1: 51)$ & $\mathrm{HD}^{\mathrm{c}}(1: 80)$ \\
\hline hip transmission & differential $(1: 2.5)$ & direct $(1: 1)$ \\
\hline knee gearbox & Maxon GP42 ${ }^{\mathrm{b}}(1: 26)$ & $\mathrm{HD}^{\mathrm{c}}(1: 80)$ \\
\hline knee transmission & cable pulley ${ }^{\mathrm{d}}(1: 3.3)$ & chain $(17: 19)$ \\
\hline hip stiffness & nonlinear $>100 \mathrm{Nm} / \mathrm{rad}$ & $\mathrm{Nm} / \mathrm{rad}$ \\
\hline knee stiffness & $\mathrm{Nm} / \mathrm{rad}$ & $\mathrm{Nm} / \mathrm{rad}$ \\
\hline \multicolumn{3}{|c|}{ sensors } \\
\hline spring deflection & \multirow{2}{*}{\multicolumn{2}{|c|}{ AVAGO AEDA3300 (80'000 qcturn) }} \\
\hline abs. joint position & Contelec WAL300 Potentiometer & \\
\hline
\end{tabular}

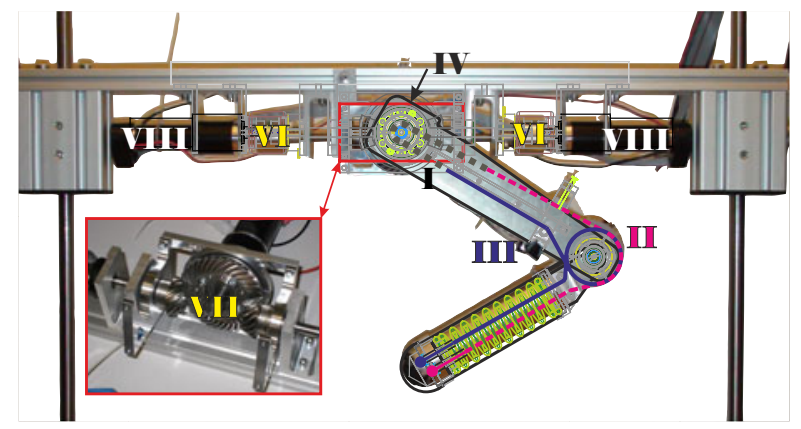

Fig. 2. FerrETH is driven by a differential gearbox in combination with SDA actuators at the hip joint and a high compliant SEA in the knee.

chain-drive (I) and two cable-pulleys (II,III) to a highly compliant spring $(\mathrm{V}, 12 \mathrm{Nm} / \mathrm{rad})$ placed in the shank. This spring creates a large compliance in the knee joint and allows for temporary energy storage during ground contact. It dominates the natural dynamics of the entire leg. The passive deflection of this spring is measured using a high-resolution incremental encoder in the knee (AVAGO AEDA3300 optical incremental encoder with $80000 \mathrm{qc} / \mathrm{turn}$ ). Absolute potentiometers (Contelec Wal300) are used to initialize the motor encoders on the remaining axes.

During running, most of the energy is being stored in the knee joint which hence undergoes substantial passive deflections. The hip actuators, on the other hand, are primarily used for precise foot positioning during the flight phase and do not store any energy. High-performance elastomer bushings (VI) are thus mounted in series with the hip motors (VIII) and form an SDA that protects the gearbox from impacts while passively suppressing undesired joint deflections.

The overall design benefits from the fact that both hip motors can be rigidly attached to the main body and that the knee 


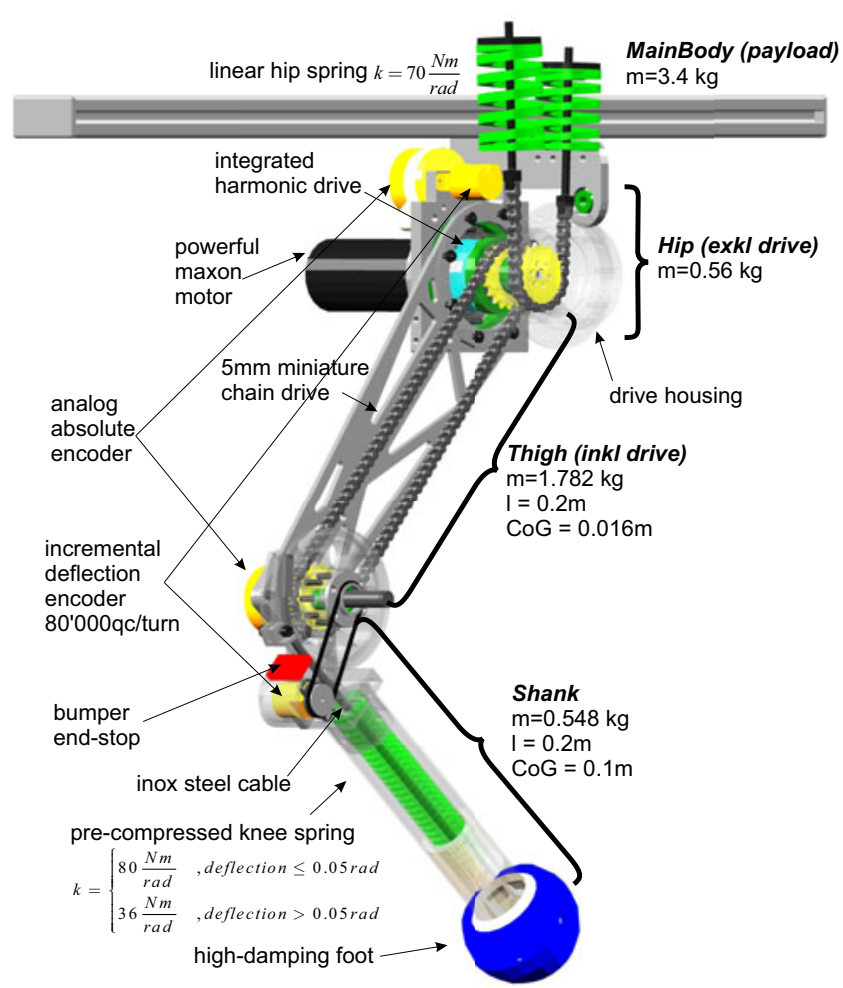

Fig. 3. Detailed system description of ScarlETH including a visualization of the SEA principle for hip a knee flexion/extension.

motor is positioned very close to the center of rotation of the hip joint. This simplifies cabling to a large extent, allows for a better protection of all actuators, centralizes the mass near the main body, and achieves a large range of motion. Hip adduction/abduction range is $\left[+90,-45^{\circ}\right]$, hip flexion/extension $\pm 75^{\circ}$, and knee flexion/extension $+20,-170^{\circ}$ from the vertical configuration. From a mechanical point of view, the main drawback of this design is the additional weight of the massive differential drive and the undesired play in the bevel gears and the planetary gearboxes of the motors.

\section{B. ScarlETH}

To avoid coupling effects and reduce the overall weight, the differential drive concept is not repeated in ScarlETH (see Fig. 3). To keep the leg inertia small, a single, weight-optimized drive unit containing harmonic drive gearboxes and two Maxon RE35 90-W DC motors for hip and knee flexion/extension serves as the hip joint axis. Improved from the previous design, a more robust chain drive and steel cable pulley connect the knee motor to a large compression spring in the shank. The same absolute potentiometers and incremental deflection encoders as in FerrETH are used in all joints to give precise state measurements at all time.

A major design difference in comparison to the FerrETH leg is the actuation of the hip joints. Instead of very stiff and highly damped elastomers, steel springs are used for SEA. Since they show nearly no passive damping, this design choice entails a fundamental shift from position/velocity control on joint level to a fully torque controlled approach. This requires adequate

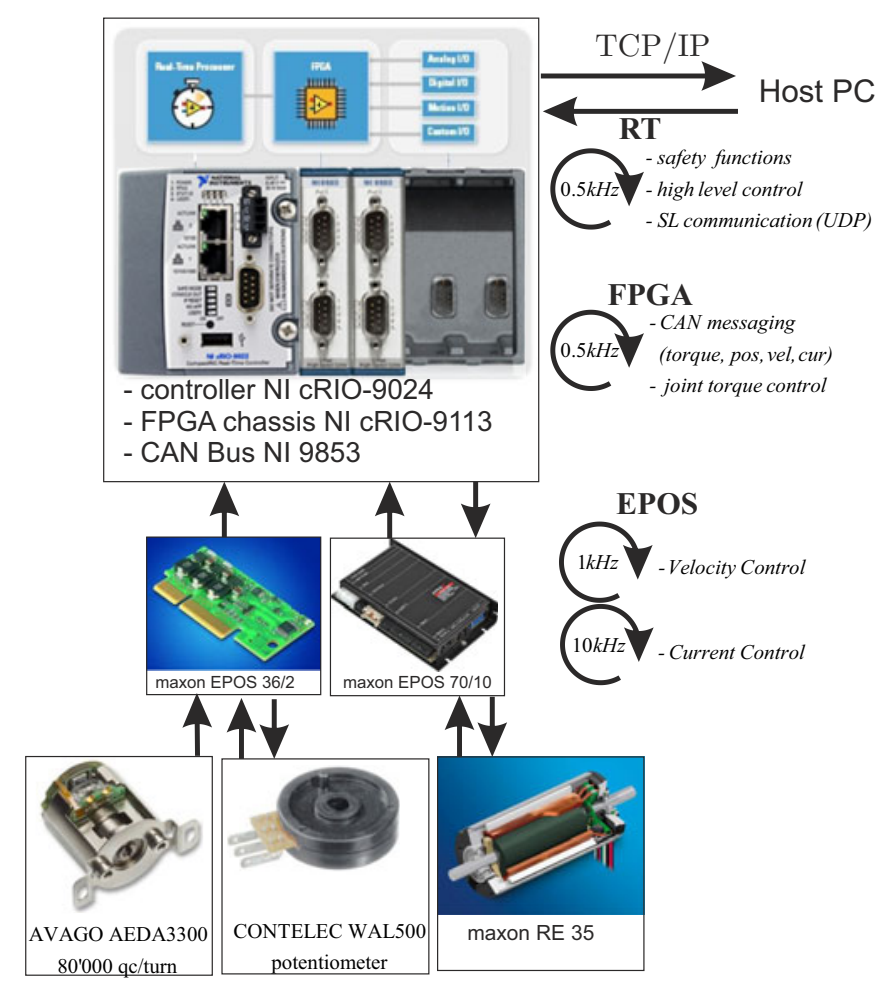

Fig. 4. Electronic control setup for FerrETH and ScarlETH is based on an NI compactRIO (RT) in combination with Maxon EPOS motor controllers.

sensing technologies and a completely backlash free design with precompressed joint springs and harmonic drive gearboxes.

This design has additional advantages: due to the high integration, a high power-to-weight ratio is achieved for the overall robot. The compact design with the chain and cable pulley transmission results in large mobility for all joints. The leg can be completely extended and retracted (knee joint $\left[-175^{\circ},+60^{\circ}\right]$ ), while the hip joint can undergo a swing angle of $\left[-80^{\circ},+80^{\circ}\right]$. Perfect linearity in the joint springs and very high-resolution encoders give very accurate joint torque measurements and allow for high fidelity force control.

\section{Electronic Setup}

Both legs use the same electronic setup for control, as shown schematically in Fig. 4. Position, torque, and high-level control are implemented on a National Instruments compactRIO [a realtime system combined with a field-programmable gate array (FPGA)], which is connected via CAN bus to a number of Maxon EPOS-modules. They are used as sensor boards to read out the encoder values and for motor control running the low level control loops for velocity and current regulation. Due to payload restrictions, these control electronics are stored offboard in the FerrETH platform.

\section{Elastic Actuator Design}

The goals of versatility, speed, and efficiency seem to be contradictory when it comes to actuation and control [21]. To make a hopping or running motion efficient, the mechanical damping 
of the elasticities in the system needs to be kept as small as possible to allow efficient energy storage when the leg is loaded during stance. However, low-damped elasticities can create undesired deflections and oscillations that make fast and precise foot positioning very difficult when the leg is unloaded during flight. This "damping dilemma" (high damping and no oscillations during flight, low damping and efficient energy storage during stance [22]) requires novel solutions based on hand-inhand integration of hardware and controller design. In this section, different concepts based on internal collisions, nonlinear damping, and active damping are presented and compared.

\section{A. Knee Actuation}

For the two systems, the oscillation frequencies of the unloaded shank segment (i.e., during flight) can be computed as

$$
f=\frac{1}{2 \pi} \sqrt{\frac{k}{m_{S} s_{S}^{2}+\theta_{S}}}= \begin{cases}10 \mathrm{~Hz} & (\text { FerrETH }) \\ 12 \mathrm{~Hz} & (\text { ScarlETH }) .\end{cases}
$$

With these high eigenfrequencies, damping of the knee oscillations (during the flight phase) is very difficult to achieve through control alone. Consequently, methods for passive mechanical damping during the flight phase need to be employed. In our lightweight design, it is impossible to use additional couplings, brakes, magnetorheological systems, or other adjustable dampers [26]. Instead, we take advantage of the fact that the leg is always loaded during the stance phase and that this loading only happens in one direction.

In FerrETH, damping of the unloaded leg is achieved through internal dynamic effects of the spring. Every time, the knee is crossing the neutral position [see Fig. 5(a)], the direction of motion of the unidirectional spring must change. This results in an internal mechanical collision within the spring, energy is lost, and the motion is slowed down. Since this only happens when the knee is unloaded, energy storage during stance is unaffected by this process. In [22], we identified two effects that contribute to increased damping during flight phase.

First, the internal collisions lead to an instantaneous reduction in joint deflection speed $\dot{\delta}\left(^{-}=\right.$before collision, ${ }^{+}=$after collision), which can be expressed as a function of the total shank inertia $\bar{\theta}_{S}=\theta_{S}+m_{S} s_{S}^{2}+m_{F} s_{F}^{2}$, the spring mass $m_{F}$, the corresponding joint distance $s_{F}$, and the pulley radius $r$ according to

$$
\frac{\dot{\delta}^{+}}{\dot{\delta}^{-}}=\frac{\bar{\theta}_{S}-m_{F} r^{2}}{\bar{\theta}_{S}+m_{F} r^{2}}=\epsilon .
$$

In a full period, the spring undergoes two collisions in the forward and backward motion. The speed over a full cycle hence scales with $\frac{\dot{\delta}_{k+1}}{\dot{\delta}_{k}}=\epsilon^{2}$. Neglecting all other energy losses in the spring (e.g., due to viscous damping or friction), this leads to a kinetic energy $T$ loss that is directly related to a decrease of the deflection amplitude $\hat{\delta}$ [see Fig. 6(a)]:

$$
\frac{T_{k+1}}{T_{k}}=\frac{\frac{1}{2} \bar{\theta}_{S} \dot{\delta}_{k+1}^{2}}{\frac{1}{2} \bar{\theta}_{S} \dot{\delta}_{k}^{2}}=\frac{\frac{1}{2} c r^{2} \hat{\delta}_{k+1}^{2}+c \bar{x}_{p} r \hat{\delta}_{k+1}}{\frac{1}{2} c r^{2} \hat{\delta}_{k}^{2}+c \bar{x}_{p} r \hat{\delta}_{k}}=\epsilon^{4} .
$$

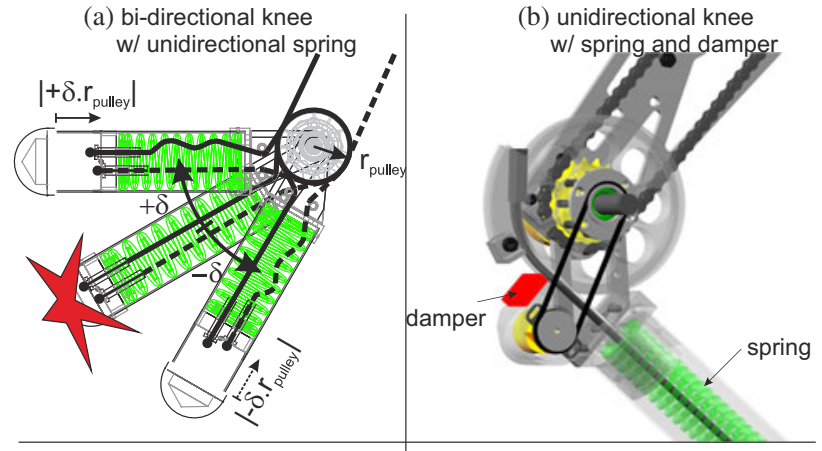

(c) pre-compressed spring

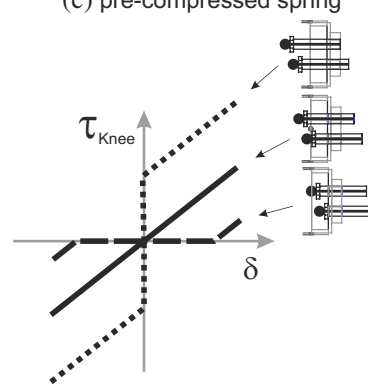

(d) pre-compressed spring-damper

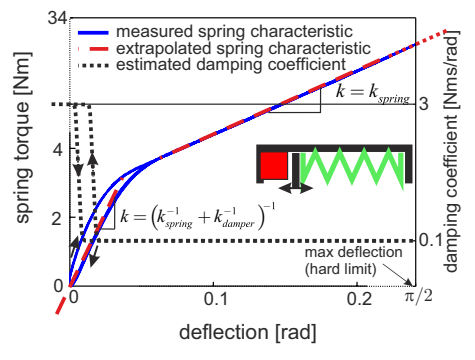

Fig. 5. Knee actuator of FerrETH exploits internal collisions (a) to increase damping during flight that can be adjusted through variable spring precompression (c). ScarlETH benefits from a nonlinear spring-damper characteristic (b), (d) that shows minimal damping and a linear, hysteresis-free torquedeflection ratio during stance (efficiency) and maximal damping with hysteresis due to the spring-damper precompression during flight phase (versatility).
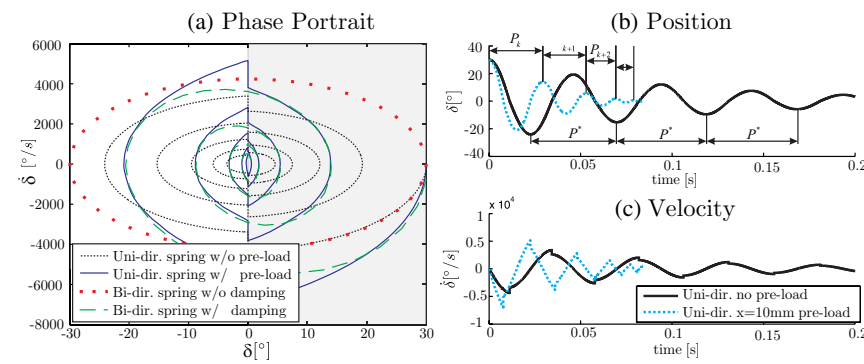

Fig. 6. Internal collisions in the knee SEA of FerrETH cause higher damping through impact losses (a) as well as a phase shortening (b).

This means the damping ratio $\frac{\hat{\delta}_{k+1}}{\hat{\delta}_{k}}$ can be adjusted within the range of $\epsilon^{4}$ and $\epsilon^{2}$ as a function of the spring precompression $\bar{x}_{p}$ and the spring stiffness $c$.

Second, the oscillation period $P$ is shortened by an increased precompression $\bar{x}_{p}$ [see Fig. 5(c)] of the spring [see Fig. 6(b)]. This can be expressed as

$$
\frac{P_{k+1}}{P_{k}}=\frac{\pi-2 \arcsin \left(\bar{x}_{p} /\left(\bar{x}_{p}+r \hat{\delta}_{k+1}\right)\right)}{\pi-2 \arcsin \left(\bar{x}_{p} /\left(\bar{x}_{p}+r \hat{\delta}_{k}\right)\right)}
$$

and additionally reduces the time until an initial deflection vanishes.

In ScarlETH, a different strategy is used that takes advantage of the fact that the knee joint during the stance phase is always loaded in the same direction [25]. We apply a combination of SEA and SDA [see Fig. 5(b)] to prevent oscillations during flight 


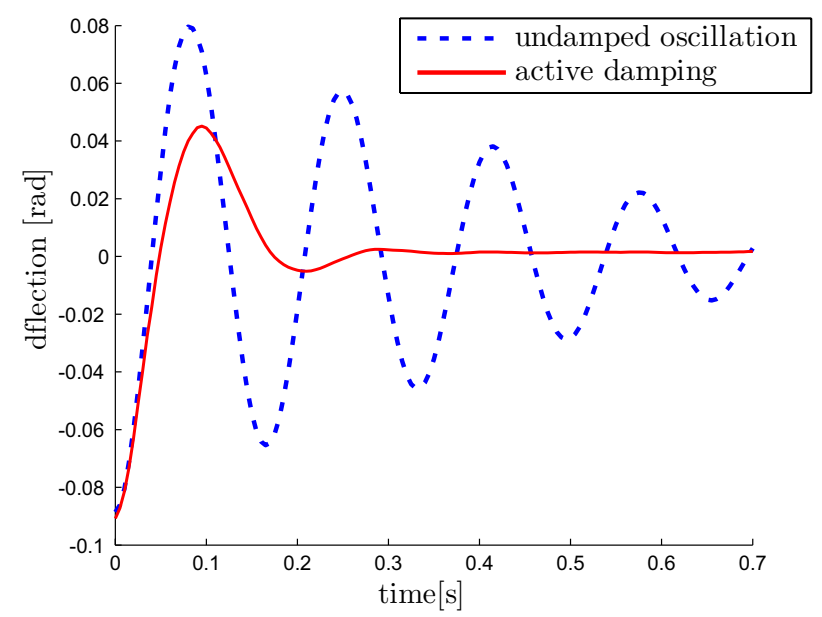

Fig. 7. Experimental results of active damping in the antagonistically precompressed hip joint of StarlETH demonstrate a highly improved behavior during flight phase.

while keeping damping low during stance. In this design, a steel spring is precompressed against a damper unit, which creates an overall nonlinear spring-damping characteristic [as shown in Fig. 5(d)]. If the leg is in contact with the ground, the actuator is continuously operating in the linear, low-damped region of the spring (SEA), allowing for efficient energy storage. During flight phase, the knee joint operates in the high-damping and high stiffness region (SDA) such that undesired deflections are immediately suppressed. In this region, additional hysteresis effects further improve position control while degrading the torque controllability. The latter is no problem, since the SEA/SDA is only operated in this region during flight phase.

\section{B. Hip Actuation}

The SDA actuation in the hip joint of FerrETH reduces the joint position control to a simple problem of motor position control. The high stiffness and strong damping in the elastomers quickly suppress any undesired deflections. The hip actuation concept of ScarlETH, on the other hand, is fundamentally different. Instead of relying on passive mechanical damping, the hip joint is designed in such a way that active damping can be achieved through force control. This requires a careful selection of the spring stiffness. Springs that are too stiff let the joint oscillate at frequencies that are impossible to control with the limited bandwidth of the actuators, while springs that are too compliant require large travel in the actuators to produce a desired force. This would decrease the force control bandwidth and drastically reduce performance.

With the chosen stiffness values (see Table I), the eigenfrequency of the hip joint motion evaluates to

$$
f=\frac{1}{2 \pi} \sqrt{\frac{k_{\mathrm{Hip}}}{\theta_{T}+m_{T} s_{T}^{2}+\theta_{S}+m_{S}\left(l_{T}+s_{S}\right)^{2}}}=6.25 \mathrm{~Hz}
$$

which can be actively damped with the hip actuator. Both the eigenfrequency of $6.25 \mathrm{~Hz}$ and the active damping performance are shown as experimental results in Fig. 7. For these plots, the hip joint was deflected by an external force and released, while the joint position was recorded using the incremental joint encoder. The controller design is discussed in more detail in Section IV-A.

\section{LOCOMOTION CONTROL}

The different joint designs presented in the previous section allow for a large variety of applications, yet they pose fundamentally different challenges in terms of control. In this section, we will focus on these challenges, present different approaches for joint position, torque, and efficient motion control, and show their applications in 1-D hopping and 2-D running. Independent of the chosen control strategy, the motors are always considered as velocity sources. A fast, low-level velocity and current controller compensates for gearbox friction and inertia effects and regulates the motor velocity. Joint position or torque control, as well as high-level running/hopping coordination, is then implemented in a cascaded structure, as is depicted in Fig. 8.

\section{A. Position Control}

High damping (FerrETH, hip), internal collisions (FerrETH, knee), and nonlinear spring-damper characteristics (ScarlETH, knee) reduce the position control problem of these joints to a trivial problem of motor position control. In contrast to that, active damping is required in the hip joint of ScarlETH since such a motor position controller implementation leads to natural joint oscillations. They are marked with a black circle in the pole-zero map of Fig. 9. The dominating and well-known spring-mass-damper dynamics expressed as a function of the motor angle $\varphi_{m}$, joint angle $\phi_{j}$, stiffness $c$, and damping $d$

$$
\theta \ddot{\phi}_{j}=c\left(\varphi_{m}-\phi_{j}\right)+d\left(\dot{\varphi}_{m}-\dot{\phi}_{j}\right)
$$

in combination with the integrator behavior of the velocity controlled motor (bandwidth $100 \mathrm{~Hz}$ [24]) allows the implementation of a linear-quadratic regulator (LQR) control structure based on joint and motor measurements (no observer required):

$\dot{\varphi}_{m}=k_{1}\left(\phi_{\mathrm{des}}-\varphi_{m}\right)-k_{2}\left(\phi_{\mathrm{des}}-\phi_{j}\right)+k_{3}\left(\dot{\phi}_{\mathrm{des}}-\dot{\phi}_{j}\right)$.

The closed-loop joint position transfer function is

$\frac{\Phi_{j}(s)}{\Phi_{\text {des }}(s)}=\frac{\left(k_{1}-k_{2}+k_{3} s\right)(c+s d)}{\theta s^{2}\left(s+k_{1}\right)+\left(s+k_{1}-k_{2}+k_{3} s\right)(c+s d)}$.

Proper adjustment of the control gains $k_{1}>k_{2}>0$ and $k_{3}>$ 0 shifts the poles to higher damped regions while keeping the response time of the system (see Fig. 9).

\section{B. Torque Control}

In StarlETH, the identified spring characteristic in both the hip and knee spring shows nearly perfect linearity with no hysteresis effects. A linear, least squares model fit shows that the springs can be used as a torque sensor with a linear regression coefficient of $R=0.999$ (hip and knee) and a mean absolute error of $\overline{|e|}_{\text {hip }}=0.08 \mathrm{Nm}$ and $\overline{|e|}_{\text {knee }}=0.05 \mathrm{Nm}$. Hence, the 


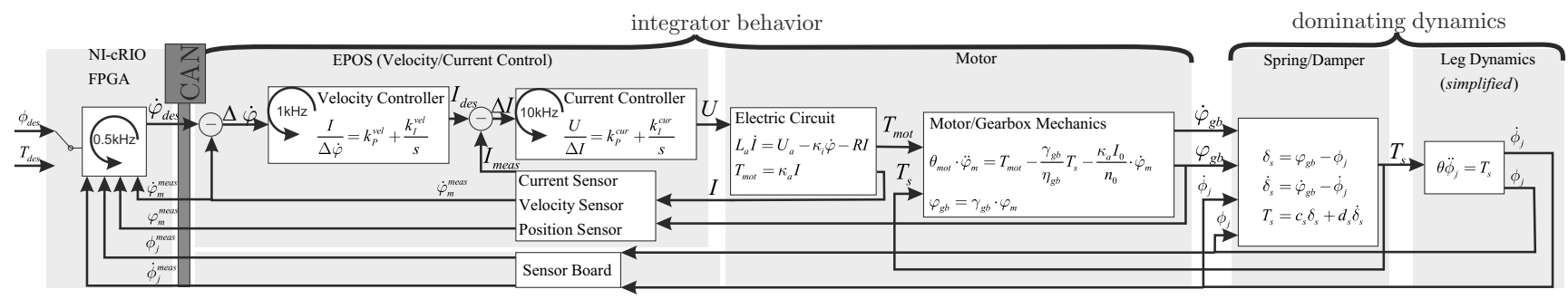

Fig. 8. Control structure is built in a cascaded structure with position/torque control implemented on the NI compactRIO device and a velocity controlled motor. The mass-spring dynamics of the leg are dominating (all other loops are much faster), allowing for large simplifications for theoretical control design.

(a) Pole-Zero Map

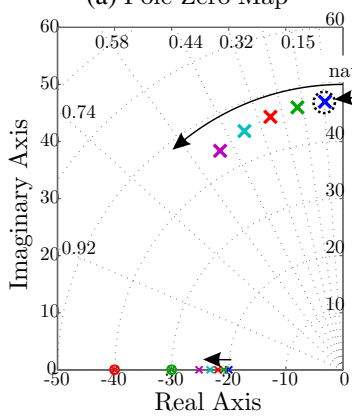

(b) Step Answer LQG

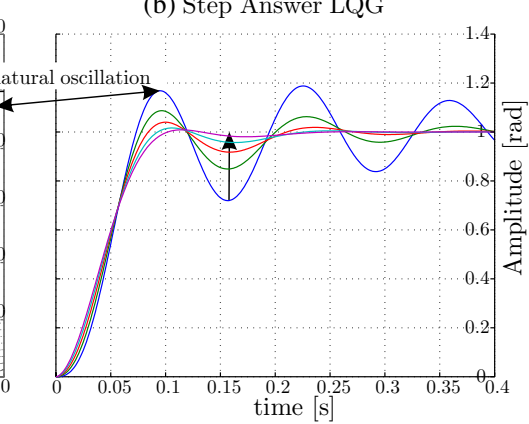

Fig. 9. LQR-based control design allows us to increase damping without sacrificing speed: the oscillating poles are shifted to higher damped regions (a) which largely improves the step response (b).

integration of additional load cells that are expensive and might break under high peak loads can be avoided, and the desired joint torques are achieved by actively regulating the spring deflections. Such a torque controller must be implemented with regard to two design criteria.

First, fast reference tracking (high closed-loop torque bandwidth in combination with high robustness) must be achieved. Fig. 10(a) shows the response to a desired step of $3 \mathrm{Nm}$ in simulation and experiment. The system can follow the reference in about $30 \mathrm{~ms}$. The closed-loop transfer function (see Fig. 10(c), blue lines) emphasizes the drawback of high compliance: with increasing amplitudes, the bandwidth substantially drops due to saturation effects. Using the complete model with the validated control parameters, the transfer function is estimated for a set of amplitudes based on a sinusoidal input. The bandwidth varies between $28 \mathrm{~Hz}$ for $1 \mathrm{Nm}$ (dashed), $15 \mathrm{~Hz}$ for $5 \mathrm{Nm}$ (dash-dotted), and $11 \mathrm{~Hz}$ for $10 \mathrm{Nm}$ (dotted). The closed-loop system is robust with a phase margin of more than $85^{\circ}$. Consequently, the resulting bandwidth is poor compared to systems with stiffer springs, yet they are still within the range of Pratt's electrical SEAs (5-25 Hz [27]) or human-assistive devices [28], and above the frequency range that is typically found in human motion (4-8 Hz [29]).

Second, next to a fast reference signal response, good disturbance rejection is crucial. This can be tested for a zero torque reference with external joint disturbance [see Fig. 10(b)]. Using a PID torque controller with low integrator gains causes substantial joint deflections (black solid line) due to the phase delay between the joint and motor angles [24]. As depicted in Fig. 10(c), the sensitivity of the PID controller (red-dashed line) has a flat ascent $(20 \mathrm{~dB} / \mathrm{dec})$ that would require higher integral
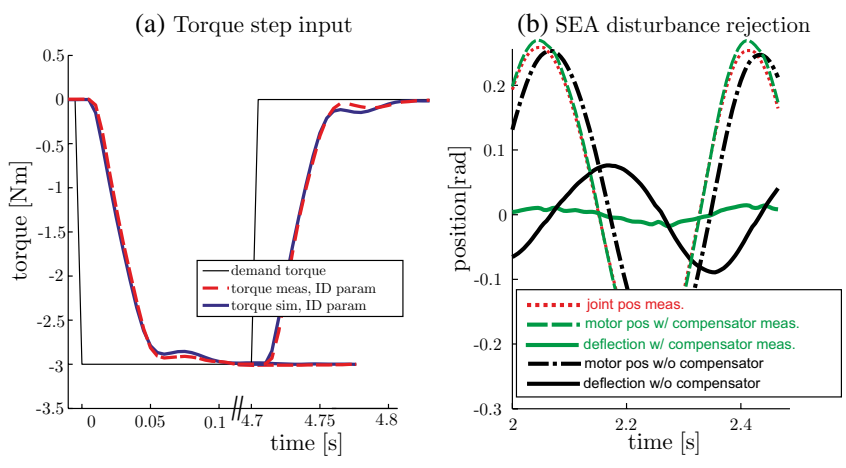

(c) Transfer estimation

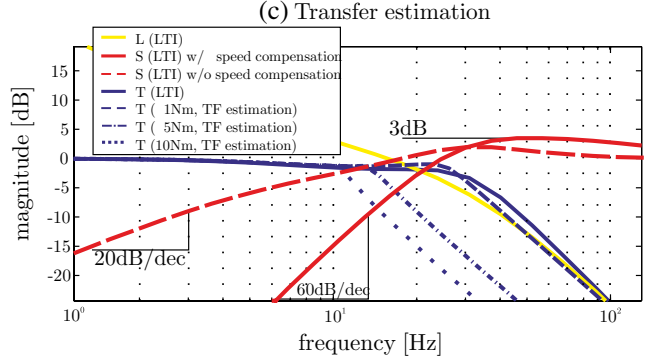

Fig. 10. Experimental and simulated results of the SEA torque controller show a fast step answer (a) with no overshoot. Using an additional disturbance compensator based on joint speed measurements allows us to significantly reduce the spring deflections when externally disturbed (b). The transfer function estimation (c) shows a bandwidth of up to $28 \mathrm{~Hz}$ for small amplitudes; about $11 \mathrm{~Hz}$ remain when saturation effects occur. Due to very small integrator gains, the overshoot is kept small $\left(T_{\max }<1 \mathrm{~dB}\right)$ and good robustness with a phase margin of more than $85^{\circ}$ is achieved. The feedforward speed compensator allows us to largely improve the sensitivity to a $60 \mathrm{~dB} / \mathrm{dec}$ ascend.

gains to keep the torque ripple small [30]. Unfortunately, this degrades the reference following behavior, having a negative influence on the robustness as well as an increased overshoot. Hence, a disturbance compensator is implemented that has no influence on the properties of the closed-loop controller. The low-pass behavior of the velocity controlled motor and the precise joint speed estimation (minimal discretization noise) allows using the joint velocity input directly as a feedforward path. This inherently ensures that the output value is independent of the input value for the stationary case since $\dot{\varphi}_{m}(s=0)=\dot{\phi}_{j}(s=0)$. The sensitivity is still bounded by $3 \mathrm{~dB}$ but shows a much better behavior for lower frequencies (at which the system is usually operated) with a slope of $60 \mathrm{~dB} / \mathrm{dec}$ (see Fig. 10(c), red solid line). The zero torque behavior successfully demonstrates this improved performance with highly reduced joint deflections (see Fig. 10(b), green solid line). At this point, the high compliance clearly helps, since the time constant for the required reaction is lower than in a system using stiff springs. 


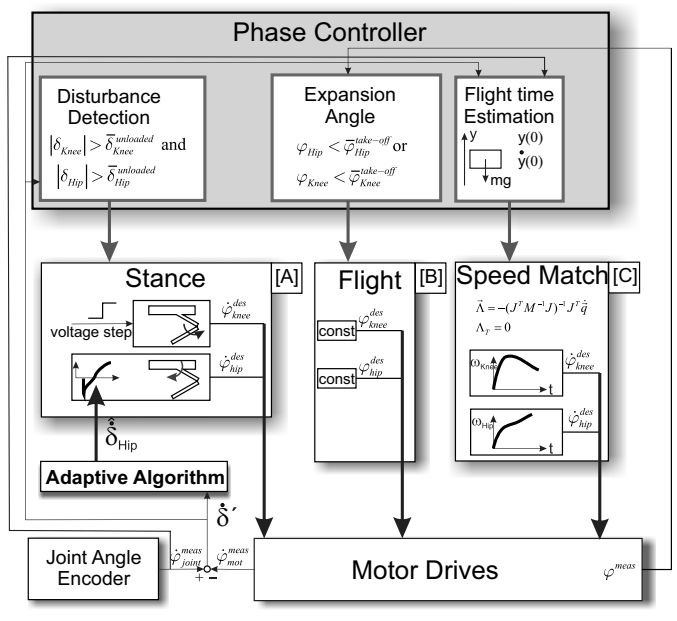

Fig. 11. Three-pronged control algorithm [23] is implemented for online adaptation of the hip joint profiles to ensure stable hopping in place.

\section{Exploiting Natural Dynamics}

The primary goal of the presented leg designs is to allow for the exploitation of natural dynamic motions. This means excess kinetic energy should be stored in the elastic elements and recovered when needed. Actuators should avoid performing negative work and merely compensate for energy losses due to damping and impact collisions. In this context, the immediate question arises how such an efficient behavior can be achieved in terms of control. This is not a trivial problem. Similar to animals, which employ different gaits for different running speeds, the actuator inputs for a robot that optimally exploit the natural dynamics of the system might need to change as a function of locomotion speed or other parameters [31]. One possibility to create motor trajectories that excite periodic oscillations for running motions is by using optimal control techniques [32] in simulation and transferring the results to the actual robot. However, since these methods require very accurate models of the hardware, it is difficult to directly transfer the results to an actual system. In the following, we thus want to present two more direct approaches to this problem.

1) Adaptive Trajectory Control: In [23], we implement a three-pronged adaptive control algorithm (see Fig. 11) that takes full advantage of the presented joint design. During stance phase, the knee motor is thrust with a desired speed profile $\dot{\phi}_{\mathrm{knee}}^{\text {des }}(t)>0$ to inject energy into the system. This ensures that during the stance phase, the knee motor never performs negative work. At the same time, the hip motor is controlled to ensure a purely vertical motion of the main body and to prevent slippage of the contact point. Starting from an initial analytical estimation of the joint trajectories based on simulations, the hip profile is adapted over a series of jumps to match the nonslippage constraint. In [23], it is shown that the algorithm converges within three successive steps.

2) Running Based on Virtual Model Control: An intuitive approach for the control of single-legged running emerges directly from biomechanical studies showing that a complex running behavior can be described to a large extent by very simple spring-mass models [11], [12]. During flight phase, the foot point is positioned to keep balance; during stance phase, energy is introduced to shape the running motion-an approach that was already implemented in the early Raibert hoppers at the MIT leg lab [10]. In ScarlETH, these control strategies can be applied directly.

First, highly damped position control allows fast foot positioning during flight phase. According to [10], the angle of attack is adapted as a function of the horizontal position $x$ and speed $\dot{x}$, while an offset angle $\alpha_{0}$ compensates for the horizontal impacts due to the articulated design:

$$
\alpha(x, \dot{x})=k_{\mathrm{FF}} \dot{x}+k_{\mathrm{FB}}\left(k_{\mathrm{Pos}}\left(x_{\mathrm{des}}-x\right)-\dot{x}\right)+\alpha_{0}
$$

whereby $k_{\mathrm{FF}}>0$ corresponds to the feedforward, $k_{\mathrm{FB}}>0$ the feedback, and $k_{\text {Pos }}>0$ to the position gain.

Second, precise torque control allows the emulation of different spring-damper behaviors between the ground contact point and the CoG of the leg through a virtual force $\mathbf{F}_{\text {virt }}$. Based on Jacobi-transposed mapping, the desired forces in Cartesian space can be mapped into joint coordinates $\mathbf{q}_{j}$ such that they create the same effect as the virtual force element [33]

$$
\begin{aligned}
\mathbf{T} & =\mathbf{J}^{T} \mathbf{F}_{\text {virt }} \\
\mathbf{J} & =\frac{\partial \mathbf{r}_{\text {Hip }}-\partial \mathbf{r}_{\text {Foot }}}{\partial \mathbf{q}_{j}} .
\end{aligned}
$$

The SLIP behavior implies a vertical spring-damper force according to

$$
F_{\text {virt }}^{y}=c_{y}\left(y_{0}-\left(y_{\text {Hip }}-y_{\text {Foot }}\right)\right)+d_{y}\left(-\dot{y}_{\text {Hip }}\right)+g_{y}
$$

with the desired spring stiffness $c_{y}$, damping coefficient $d_{y}$, and gravity compensation term $g_{y}$. Due to bandwidth limitation of the actuator, the virtual stiffness (as well as the damping coefficient) is bounded by $c_{y}^{\max } \approx \omega_{\mathrm{BW}}^{2} m_{\mathrm{Hip}}$ (actuator bandwidth $\omega_{\mathrm{BW}}$ and hip mass $m_{\mathrm{Hip}}$ ). To nevertheless ensure efficient excitation, we additionally constrained the knee joint motion such that the actuator always introduces energy into the system $P_{\text {knee }}>0$. In terms of velocity, this can be expressed as $\dot{\varphi}_{m}>0$, since throughout stance the torque at the knee joint is always positive.

To control the forward running motion, the horizontal force is modulated based on a simple proportional position controller

$$
F_{\text {virt }}^{x}=\operatorname{sat}\left(k_{x}\left(x_{\text {des }}-x_{\text {base }}\right)\right) \in\left[-F_{\text {neg }}^{x}, F_{\text {neg }}^{x}\right] .
$$

To avoid tipping over or slipping, the force is limited by a saturation function sat ().

\section{RESULTS}

Both systems were tested thoroughly in 1-D and 2-D hopping experiments. Therefore, we constructed a test bench that consists of a planar guiding unit connected to the main body. The entire system can freely move in horizontal and vertical direction on a rolling unit with low friction. Cable pulley encoders in both directions are used to measure the absolute position of the main body. Fig. 12 shows the knee joint and motor position during the stance phase of FerrETH(a) in 1-D hopping based on the trajectory adaptation algorithm (see Section IV-C1), respectively, of ScarlETH(b) in 2-D virtual model hopping (see 

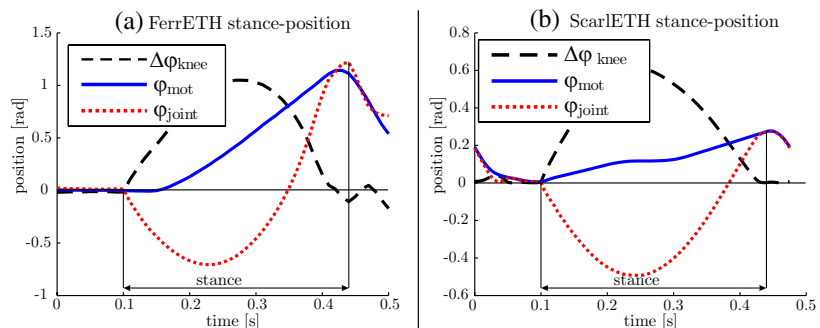

(c) FerrETH stance-power
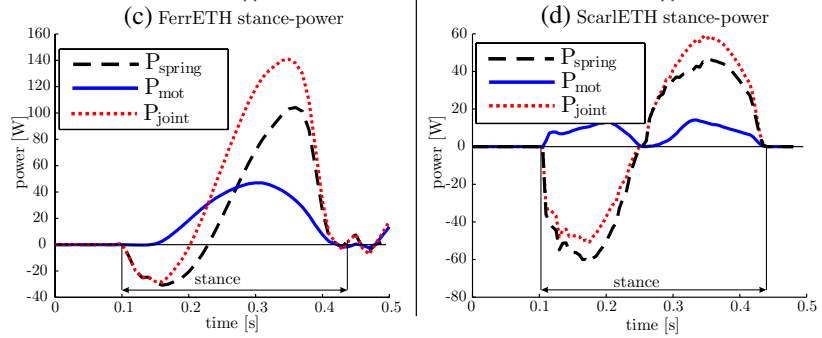

Fig. 12. Experimental results of hopping in place with FerrETH and ScarlETH indicate the benefit of the large compliance in the knee joint: (a) and (b) Motor travel distance (and its speed) remains only a fractional part of the joint motion. (c) and (d) Motor introduces energy during the entire stance phase with small peak power and zero negative work.

Section IV-C2). As expected, the spring compliance ensures that the travel distance of the motor is much smaller than the actual joint motion. Furthermore, the motor is always delivering positive power [see Fig. 12(c) and (d)]. The spring stores kinetic energy after impact and returns it before liftoff. This is in stark contrast to a stiff actuation approach in which breaking and accelerating energy needs to be provided by the actuator. To be able to compare the mechanical efficiency of energy storage in single-legged hopping (among robots but also in comparison to values found for human or animal in-place hopping), we define the ratio between the positive work performed by the actuator and the positive joint work as the hopping efficiency $\eta$

$$
\eta=1-\frac{\int_{T} \max \left(P_{\text {mot }}, 0\right) d t}{\int_{T} \max \left(P_{\text {joint }}, 0\right) d t} .
$$

Since electric energy recuperation is generally not possible, we use the notation $\max (P, 0)$. Hence, (14) indicates how much of the positive mechanical work in a hopping cycle $\left(\int_{T} \max \left(P_{\text {joint }}, 0\right) d t\right)$ can be provided passively. To keep the efficiency maximal, the actuator should never perform negative work. Additionally to the efficiency coefficient $\eta$, the maximal joint speed in relation to the maximal motor speed $\omega_{\mathrm{rel}}=\frac{\dot{\phi}_{\mathrm{joint}}}{\dot{\varphi}_{\mathrm{mot}}}$, as well as the maximal joint power in relation to the maximal motor power $P_{\text {rel }}=\frac{P_{\text {joint }}}{P_{\text {mot }}}$ clearly indicate the benefit of highly compliant SEA

\begin{tabular}{l|cc} 
& FerrETH & ScarlETH \\
\hline$\eta$ & 0.33 & 0.64 \\
$\omega_{\text {rel }}$ & 3.9 & 4.7 \\
$P_{\text {rel }}$ & 2.2 & 4.1
\end{tabular}

Both robotic devices have an excellent mechanical efficiency. Since energy can be introduced to the system throughout the entire stance phase (not only during the acceleration phase), less power is required for the same amount of work $\left(P_{\text {rel }}>1\right)$, and the motors can hence be reduced in size and weight. In both

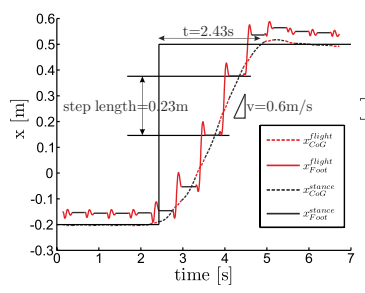

(a)

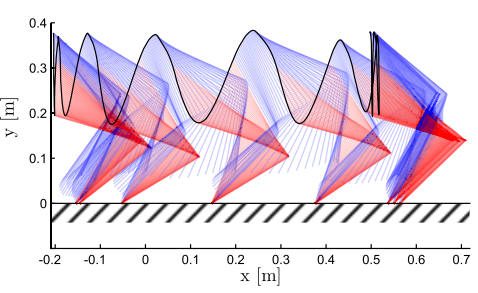

(b)
Fig. 13. Two-dimensional running experiments were successfully conducted with ScarlETH based on virtual model control. (a) target position step input. (b) running configuration in experiment.

\begin{tabular}{l|cc} 
& FerrETH & ScarlETH \\
\hline$\eta$ & 0.33 & 0.64 \\
$\omega_{\text {rel }}$ & 3.9 & 4.7 \\
Prel & 2.2 & 4.1
\end{tabular}

experiments, the joint springs lead to a large amplification of the output speed $\left(\omega_{\text {rel }}>1\right)$.

The controllability of ScarlETH is superior in comparison to FerrETH. Hopping height can be modified by changing the neutral point of the virtual spring element (12), while transition motions (e.g., from rest to hop or vice versa) or changes in the hopping frequency result from a very intuitive change of the virtual damping $d_{y}$, stiffness $c_{y}$ parameters, or offset force $g_{y}$. The virtual forces in the horizontal direction (13) in combination with the angle of attack control (9) allows us to reach arbitrary goal positions without any overshoot (see Fig. 13), whereby step lengths of about $0.25 \mathrm{~m}$ and top speeds of about $0.6 \mathrm{~m} / \mathrm{s}$ are achieved [25]. The mechanical cost of transport in this experiment with energy expenditure defined as the integral of positive mechanical motor power is about COT $=0.9$, which is rather low in comparison with robotic devices of similar weight [21].

FerrETH is rather hard to control: changes in the desired hopping height always require a new adaptation of the hip motion profile [23], [34]. Reactions against uneven ground or other external disturbances are hence hard to achieve with this type of adaptive control.

\section{CONCLUSION}

This paper has summarized the design and control of two robotic legs (FerrETH and ScarlETH) that were developed at our lab in order to combine versatility, speed, and efficiency in one single piece of hardware. We highlighted the various possibilities that these legs offer with respect to position/torque control as well as the exploitation of natural dynamic motions, and compared results for periodic hopping and running motions. Both legs are intended for integration in a quadruped robot that is able to robustly perform highly dynamic maneuvers and that shows large mobility in all joints. For the mechanical design, this enforced a very tight integration of all components (especially the springs), a lightweight construction with all actuators at the hip joint, and powerful motors.

Inspired by nature, where elastic elements in muscles and tendons largely contribute to an efficient running motion, we include large compliances in our system that not only protect the motors from impacts but also allow the temporary storage of energy. Internal collisions and nonlinear spring damper 
characteristics in the knee joint mechanically solve the damping dilemma: during the contact phase (when the joints are only deflected in one direction), very low mechanical damping ensures an efficient energy storage. As soon as the leg leaves the ground, it passes the neutral position which either leads to internal collisions (FerrETH) or to very high mechanical damping $(\mathrm{ScarlETH})$ and hence to a fast/immediate decrease of the undesired deflections. The two systems have one fundamental difference: In FerrETH, the hip joint remains position controlled based on high damping. This allows very accurate and fast joint position control, while at the same time, the motors are protected from impact loads. In contrast, the hip of ScarlETH is built in a very compliant way that allow for precise torque control. Hand-in-hand design of the mechanical part and joint control facilitates active damping of the deflection oscillations during noncontact phases through an LQR control setup.

Although both systems demonstrated their applicability for planar running with similar performance with regard to system efficiency, ScarlETH has a number of beneficial properties that favor it for future locomotion studies. The greatest advantage is its full torque controllability. In contrast to a complicated and restricted trajectory adaptation algorithm [23] that is necessary due to the missing precise torque controllability in the SDA hip joint, well-studied and advanced (model-based) torque control policies [33], [35]-[37] can be implemented. These very generic policies make the robot more versatile and robust, especially when it comes to complicated multilegged systems. Hence, having with ScarlETH a system that combines full torque and position control capability in one single device, this leg will be used in a quadruped running and climbing machine.

\section{REFERENCES}

[1] G. A. Cavagna, N. C. Heglund, and C. R. Taylor, "Mechanical work in terrestrial locomotion: Two basic mechanisms for minimizing energy expenditure," AJP Regul. Integr. Comp. Physiol., vol. 233, no. 5, pp. 243-261, 1977.

[2] M. H. Dickinson, C. T. Farley, R. J. Full, M. A. R. Koehl, R. Kram, and S. Lehman, "How animals move: An integrative view," Science, vol. 288, no. 5463 , pp. $100-106,2000$.

[3] K. Hirai, "Current and future perspective of Honda humamoid robot," in Proc. IEEE/RSJ Int. Conf. Intell. Robots Syst., Sep. 1997, pp. 500-508.

[4] J. Yamaguchi, S. Inoue, D. Nishino, and A. Takanishi, "Development of a bipedal humanoid robot having antagonistic driven joints and three DOF trunk," in Proc. IEEE/RSJ Int. Conf. Intell. Robots Syst., Oct. 1998, pp. 96-101.

[5] J. Estremera and P. Gonzalez de Santos, "Generating continuous free crab gaits for quadruped robots on irregular terrain," IEEE Trans. Robot. Autom., vol. 21, no. 6, pp. 1067-1076, Dec. 2005.

[6] M. Kalakrishnan, J. Buchli, P. Pastor, M. Mistry, and S. Schaal, "Fast, robust quadruped locomotion over challenging terrain," in Proc. IEEE Int. Conf. Robot. Autom., May 2010, pp. 2665-2670.

[7] K. Byl, K. Shkolnik, S. Prentice, N. Roy, and R. Tedrake, "Reliable dynamic motions for a stiff quadruped," in Proc. 11th Int. Symp. Exp. Robot., 2009, pp. 319-328.

[8] C. D. Remy, O. Baur, M. Latta, A. Lauber, M. Hutter, M. H. Hoepflinger, C. Pradalier, and R. Siegwart, "Walking and crawling with ALoF: A robot for autonomous locomotion on four legs," Ind. Robot: An Int. J., vol. 38, no. 3, pp. 264-268, 2011.

[9] M. H. Hoepflinger, C. D. Remy, M. Hutter, and R. Siegwart, "Haptic terrain classification on natural terrains for legged robots," in Proc. Int. Conf. Climbing Walking Robots, 2010, pp. 785-792.

[10] M. H. Raibert, Legged Robots that Balance, (ser. The MIT Press Series in Artificial Intelligence). Cambridge, MA: MIT Press, 1986.
[11] R. M. Alexander, "3 uses for springs in legged locomotion," Int. J. Robot. Res., vol. 9, no. 2, pp. 53-61, 1990.

[12] R. Blickhan, "The spring-mass model for running and hopping," $J$. Biomech., vol. 22, no. 11/12, pp. 1217-1227, 1989.

[13] P. Gregorio, M. Ahmadi, and M. Buehler, "Experiments with an electrically actuated planar hopping robot," in Proc. 3rd Int. Symp. Exp. Robot. III, 1994, pp. 269-281.

[14] H. D. Taghirad, "Analysis, design, and control of hopping robot" M.Eng. Project Rep., Dept. Mech. Eng., McGill Univ., Montreal, QC, Canada, 1993.

[15] P. Gregorio, M. Ahmadi, and M. Buehler, "Design, control, and energetics of an electrically actuated legged robot," IEEE Trans. Syst. Man Cybern. B, Cybern., vol. 27, no. 4, pp. 626-634, Aug. 1997.

[16] L. R. Palmer, D. E. Orin, D. W. Marhefka, J. P. Schmiedeler, and K. J. Waldron, "Intelligent control of an experimental articulated leg for a galloping machine," in Proc. IEEE Int. Conf. Robot. Autom., Sep. 2003, pp. 3821-3827.

[17] H. De Man, D. Lefeber, and J. Vermeulen, Design and Control of a Robot with One Articulated Leg for Locomotion on Irregular Terrain. ch. VI, New York: Springer, 1998, vol. 405, pp. 417-424.

[18] S. Curran and D. E. Orin, "Evolution of a jump in an articulated leg with series-elastic actuation," in Proc. IEEE Int. Conf. Robot. Autom., May 2008, pp. 252-258.

[19] J. W. Hurst, "The role and implementation of compliance in legged locomotion" Ph.D. dissertation, Carnegie Mellon Univ., Pittsburgh, PA, 2008.

[20] J. G. Nichol and K. J. Waldron, "Biomimetic leg design for untethered quadruped gallop," in Proc. Int. Conf. Climbing Walking Robots, 2002, pp. $49-54$.

[21] A. D. Kuo, "Choosing your steps carefully: Trade-offs between economy and versatility in dynamic walking bipedal robots," IEEE Robot. Autom. Mag., vol. 14, no. 2, pp. 18-29, Jun. 2007.

[22] M. Hutter, C. D. Remy, and R. Siegwart, "Design of an articulated robotic leg with nonlinear series elastic actuation," in Proc. Int. Conf. Climbing Walking Robots, 2009, pp. 645-652.

[23] M. Hutter, C. D. Remy, and R. Siegwart, "Adaptive control strategies for open-loop dynamic hopping," in Proc. IEEE/RSJ Int. Conf. Intell. Robots Syst., Oct. 2009, pp. 154-159.

[24] M. Hutter, C. D. Remy, M. H. Hoepflinger, and R. Siegwart, "High compliant series elastic actuation for the robotic leg ScarlETH," in Proc. Int. Conf. Climbing Walking Robots, 2011, pp. 507-514.

[25] M. Hutter, C. D. Remy, M. H. Hoepflinger, and R. Y. Siegwart, "ScarlETH: Design and control of a planar running robot," in Proc. IEEE/RSJ Int. Conf. Intell. Robots Syst., Sep. 2011, pp. 562-567.

[26] F. P. Boyle, N. K. Petek, and D. P. Smith, "Method for controlling motion using an adjustable damper," U.S. Patent 5582 385, Dec. 1996.

[27] J. E. Pratt and B. T. Krupp, "Series elastic actuators for legged robots," Proc. SPIE, Unmanned Ground Veh. Technol. VI, vol. 5422, pp. 135-144, 2004.

[28] K. Kong, J. Bae, and M. Tomizuka, "A compact rotary series elastic actuator for human assistive systems," IEEE/ASME Trans. Mechatronics, vol. 17, no. 2, pp. 288-297, Apr. 2012.

[29] K. Kyoungchul, B. Joonbum, and M. Tomizuka, "Control of rotary series elastic actuator for ideal force-mode actuation in human and robot interaction applications," IEEE/ASME Trans. Mechatronics, vol. 14, no. 1, pp. 105-118, Feb. 2009.

[30] H. Vallery, R. Ekkelenkamp, H. van der Kooij, and M. Buss, "Passive and accurate torque control of series elastic actuators," in IEEE/RSJ Int. Conf. Intell. Robots Syst., Oct./Nov. 2007, pp. 3534 3538.

[31] C. D. Remy, K. W. Buffinton, and R. Y. Siegwart, "Energetics of passivity based running with high-compliance series elastic actuation," Int. J. Mechatronics Manuf. Syst., vol. 5, pp. 120-134, 2012.

[32] C. D. Remy, K. W. Buffinton, and R. Siegwart, "A Matlab framework for efficient gait creation," in Proc. IEEE/RSJ Int. Conf. Intell. Robots Syst., Sep. 2011, pp. 190-196.

[33] J. Pratt, C. M. Chew, A. Torres, P. Dilworth, and G. Pratt, "Virtual model control: An intuitive approach for bipedal locomotion," Int. J. Robot. Res., vol. 20, no. 2, pp. 129-143, 2001.

[34] M. Hutter, "Hopping with an articulated robotic leg" Master's thesis, ETH Zurich, Zurich, Switzerland, 2009.

[35] L. Sentis, Compliant Control of Whole-Body Multi-Contact Behaviors in Humanoid Robots. London, U.K.: Springer Global Editorial, 2009, pp. 29-66. 
[36] M. Mistry, J. Buchli, and S. Schaal, "Inverse dynamics control of floating base systems using orthogonal decomposition," in Proc. IEEE Int. Conf. Robot. Autom., May 2010, pp. 3406-3412.

[37] M. Hutter, C. D. Remy, M. H. Hoepflinger, and R. Siegwart, "Slip running with an articulated robotic leg," in Proc. IEEE/RSJ Int. Conf. Intell. Robots Syst., Oct. 2010, pp. 4934-4939.

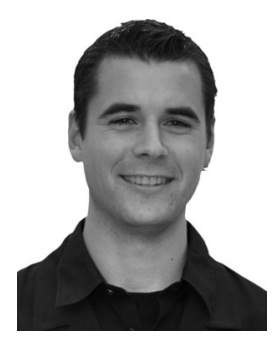

Marco Hutter was born in 1985. He received the Master's degree in mechanical engineering from the Swiss Federal Institute of Technology Zurich (ETHZ), Zurich, Switzerland, in 2009, where he is currently working toward the Ph.D. degree in the Autonomous System Laboratory.

His research interests include dynamic locomotion with quadrupeds, whereby he is working on the hardware design, low-level force and position control, as well as on model-based walking and running using passive compliance.

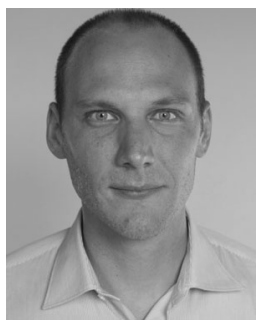

C. David Remy received the Master's degree in mechanical engineering from the University of Wisconsin, Madison, the Diploma degree in engineering cybernetics from the University of Stuttgart, Stuttgart, Germany, and the Ph.D. degree from the Swiss Federal Institute of Technology Zurich, Zurich, Switzerland.

He is currently an Assistant Professor of mechanical engineering at the University of Michigan, Ann Arbor. His research interests include the design, simulation, and control of legged and other nonlinear systems. Drawing inspiration from biology and biomechanics, he is particularly interested in the effect and exploitation of natural dynamic motions, the role of different gaits, and the possibility of force/torque controllable systems, both in conceptual models and in hardware realization.

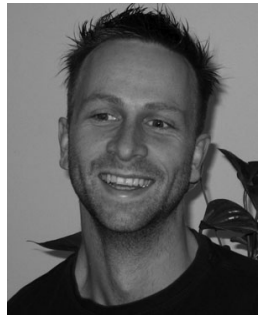

Mark A. Hoepflinger received the M.Sc. degree in electrical engineering and information technology from the Swiss Federal Institute of Technology Zurich, Zurich, Switzerland, in 2007, where he is currently working toward the Ph.D. degree at the Autonomous Systems Laboratory.

His main focus lies on research in the field of perception for walking robots.

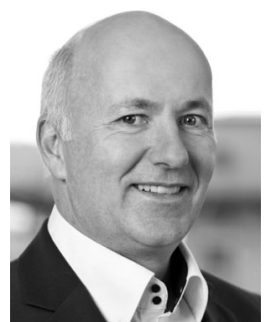

Roland Siegwart (F'08) was born in 1959. He received the Master's and Ph.D. degrees in mechanical engineering from the Swiss Federal Institute of Technology Zurich (ETH Zurich), Zurich, Switzerland.

He has been a Full Professor of Autonomous Systems and Vice President Research and Corporate Relations at ETH Zurich since 2006 and 2010, respectively. He was a Postdoctoral Fellow at Stanford University, Senior Researcher at ETH, and the R\&D Director at MECOS Traxler AG. From 1996 to 2006, he was a Professor of autonomous robots at the EPFL Lausanne and a member of the Board of Directors of the School of Engineering (during 2002-2006).

Dr. Siegwart is a member of the Swiss Academy of Engineering Sciences, and an officer of the International Federation of Robotics Research. He served as Vice President (2004-05) and AdCom Member (2007-10) of the IEEE Robotics and Automation Society. He is and was coordinator of four European projects in the field of unmanned aerial vehicles and autonomous robot navigation. $\mathrm{He}$ was the General Chair of several conferences on robotics including the 2002 IEEE/RSJ International Conference on Intelligent Robots and Systems, 2007 IEEE/ASME International Conference on Advanced Intelligent Mechatronics, 2007 6th International Conference on Field and Service Robotics, and 2009 International Symposium on Robotics Research. He is a Co-Founder of multiple successful spin-off companies specializing in robotics. 